\title{
MARQUEURS BIOCHIMIQUES DE POLLUTION DANS LES ÉCOSYSTĖMES AQUATIQUES CONTINENTAUX. EXEMPLES D'UTILISATION ET PERSPECTIVES POUR LE GESTIONNAIRE.
}

\author{
P. FLAMMARION (1), A. DEVAUX (2,3) ET J. GARRIC (1)
}

(1) CEMAGREF, Division Biologie des Ecosystèmes Aquatiques

3 bis Quai Chauveau, CP 220, 69336 Lyon cedex 9, France.

tél. : 0472208785 ; fax : 0478477875

patrick.flammarion@cemagref.fr

(2) INRA-SCRIBE, Equipe d'Ecotoxicologie Aquatique

Campus de Beaulieu, 35042 Rennes cedex, France.

(3) ENTPE, Laboratoire des Sciences de l'Environnement, rue Maurice Audin, 69518 Vaulx en Velin cedex, France.

Reçu le 2 septembre 1999

Accepté le 12 septembre 2000

Received 2 September, 1999

Accepted 12 september, 2000

\section{RÉSUMÉ}

En complément des analyses chimiques, la mesure de biomarqueurs permet de disposer d'informations sur la nature et le niveau de la contamination chimique mais aussi sur la santé des organismes vivants et des populations des écosystèmes aquatiques. Parmi les biomarqueurs, la mesure de marqueurs biochimiques chez les poissons parait particulièrement intéressante en termes de sensibilité, spécificité et précocité. En cela, les biomarqueurs viennent en complément des mesures traditionnelles de bioindication sur les populations d'invertébrés in situ. L'induction de l'activité enzymatique Ethoxy RésorufineO-Dééthylase (EROD) est le signe de l'exposition des poissons aux Hydrocarbures Aromatiques Polycycliques (HAPs), PolyChloroBiphényles coplanaires (PCBs) et dioxines, tandis que l'inhibition de l'activité AcétylCholinEstérase (AChE) traduit l'exposition à certains produits phytosanitaires (organophosphorés et carbamates). D'autre part, la mesure d'adduits à l'ADN et de cassures de brins d'ADN renseigne sur la pression génotoxique à laquelle sont exposés les poissons. La synthèse de vitellogénine chez le poisson mâle est quant à elle le signe de l'exposition des poissons à des polluants qui miment l'activité des oestrogènes, entraînant alors un impact sur la reproduction. Ces exemples de biomarqueurs, en voie de validation complète, pourraient être intégrés utilement dans un réseau pérenne de mesure en routine de la qualité et de la santé des écosystèmes aquatiques.

Mots-clés : biomarqueur, bioindicateur, EROD, acétylcholinestérase, génotoxicité, vitellogénine, biosurveillance. 


\section{BIOCHEMICAL MARKERS OF ENVIRONMENTAL CONTAMINATION IN CONTINENTAL AQUATIC ECOSYSTEMS. EXAMPLES OF USE AND PROSPECTS FOR MANAGEMENT.}

\section{ABSTRACT}

In addition to conventional chemical analyses, biomarkers are considered as useful tools for monitoring chemical contamination and assessing health of organisms and populations in aquatic ecosystems. Biochemical markers represent sensitive and early warning systems of the exposure of aquatic organisms to pollutants, thus complementing more global studies on field invertebrate populations on a long-term time scale. Among biochemical markers, induction of the enzymatic EthoxyResorufin-O-Deethylase (EROD) activity in fish is specific of exposure to Polycyclic Aromatic Hydrocarbons (PAHs), coplanar PolyChlorinated Biphenyls (PCBs) and dioxins. Moreover, inhibition of AcetylCholinEsterase (AChE) activity can indicate the response of fish to pesticides like organophosphorous compounds and carbamates. Genotoxicity assessment through the measurement of DNA adducts or DNA strand breaks gives rise to relevant information about genetic damage encountered by exposed species. Furthermore, the measure of vitellogenin synthesis in male fish has been successfully used as a biomarker of estrogenic effect of xenobiotics that leads to adverse effects on reproduction. These biomarkers should be integrated in extensive biomonitoring networks in order to complete their validation.

Key-words : biomarker, bio-indicators, EROD, acetylcholinesterase, genotoxicity, vitellogenin, biomonitoring.

\section{INTRODUCTION}

L'analyse chimique des polluants présents dans les différents compartiments des écosystèmes aquatiques n'est pas toujours possible du fait de la multiplicité des molécules présentes, et ceci souvent à des concentrations inférieures aux limites de détection analytique (NARBONNE, 1998). Par ailleurs, une telle approche ne renseigne pas sur les risques encourus par les populations animales ou végétales exposées aux polluants (LÉVÊQUE, 1997), et ne peut, à elle seule, prédire les effets biologiques des mélanges de contaminants (synergies...) ni quantifier simplement la biodisponibilité des polluants pour les organismes vivants (DUTKA, 1998). De ce fait, le gestionnaire manque d'informations sur l'urgence des mesures à prendre pour améliorer l'état de santé de ces écosystèmes (LASCOMBE, 1997), ou protéger la biodiversité et l'intégrité des écosystèmes (LÉVÊQUE, 1997).

Le suivi de la perturbation de cibles biologiques peut pallier cette difficulté. Par exemple, la spécificité d'un biomarqueur pour certaines familles de molécules chimiques (hydrocarbures aromatiques polycycliques, polychlorobiphényles, métaux lourds, produits phytosanitaires...) permet d'une part de révéler la présence de ces polluants, et d'autre part de renseigner sur la biodisponibilité de ces polluants ainsi que sur les effets biologiques précoces sur les organismes (KRAMER et BOTTERWEG, 1991 ; AMIARD et al., 1998).

L'objet de cet article n'est pas de réactualiser les très nombreuses synthèses existantes sur les biomarqueurs dans le cadre de l'évaluation de la qualité des écosystèmes (par exemple: MC CARTHY et SHUGART, 1990 ; HUGGETT et al., 1992 ; LIVINGSTONE, 1993 ; LAGADIC et al., 1998), mais, sans prétention à l'exhaustivité, de situer, par rapport à d'autres variables biologiques utilisées en routine par les gestionnaires, les apports potentiels et concrets, ainsi que les limites, de quatre marqueurs biochimiques, choisis à titre d'illustrations et susceptibles d'offrir des perspectives intéressantes. 


\section{INTÉRÊTS ET COMPLÉMENTARITÉ DES MESURES DE VARIABLES BIOLOGIQUES}

Une gestion adaptée et pertinente des milieux aquatiques doit reposer sur des outils d'évaluation de l'intégrité des écosystèmes mise à mal par le transfert et l'accumulation dans l'environnement des produits de l'activité humaine. Parallèlement aux mesures chimiques, désormais utilisées dans les programmes de surveillance, la mesure de variables biologiques est susceptible d'apporter une information intégrée sur l'état de l'écosystème aquatique ainsi que sur les phénomènes de bioaccumulation et de bioamplification (LÉVÊQUE, 1997). De ce fait, les variables biologiques sont un complément indispensable au sein d'un programme de surveillance et d'alerte (LASCOMBE, 1997).

Les variables biologiques mesurées peuvent par exemple se traduire par des indices reposant sur l'étude d'effectifs de populations. En France, l'exemple le plus connu est celui de I'IBGN, Indice Biologique Global Normalisé, indice fondé sur les familles de macroinvertébrés. Ce type de mesure fait partie des bioindicateurs qui concernent les populations ou communautés dont l'abondance et la diversité permettent de caractériser une modification du milieu. D'autres bioindicateurs concernent les oligochètes (LAFONT, 1989), les mollusques (MOUTHON, 1993) ou les diatomées (DESCY et COSTE, 1988). Si ces indices donnent une note objective sur l'état de santé des communautés, leurs valeurs sont néanmoins délicates à relier aux concentrations des contaminants, car ils sont intégrateurs d'une multitude de variables (dont l'hydrologie notamment). On peut s'intéresser également à des variables biologiques qui soient à la fois précoces et sensibles, mais aussi aptes à fonder un diagnostic sur la qualité de l'écosystème et sur la nature et le degré de contamination. L'intérêt de l'étude de ces dernières, dans une volonté d'évaluer le risque pour l'écosystème, est de prévenir des dommages écologiques irréversibles sans attendre que la probabilité d'apparition de ces dégâts soit trop forte.

C'est dans ce souci que les chercheurs se sont intéressés au développement de biomarqueurs que représentent les «indicateurs biochimiques, physiologiques ou histologiques d'exposition, ou d'effet, d'un organisme à des contaminants " (HUGGETT et al., 1992 ; BENSON et DI GIULIO, 1992). L'étendue des biomarqueurs est extrêmement vaste et concerne des mesures de réponse immunologique, de paramètres sanguins, d'effets histopathologiques, de réserves énergétiques, d'indicateurs de croissance etc. (HUGGETT et al., 1992). A titre d'exemple, les marqueurs biochimiques ont été utilisés pour détecter l'exposition à des contaminants aussi divers que les Hydrocarbures Aromatiques Polycycliques (HAPs), les PolyChloroBiphényles (PCBs), les métaux ou les produits phytosanitaires, en mesurant des activités enzymatiques spécifiques ou en détectant l'expression des gènes impliqués. Ce type de mesure, en donnant une information sur une exposition à certains contaminants et en quantifiant une réponse sublétale, est donc fondamentalement différent d'autres techniques récentes comme les kits commerciaux écotoxicologiques qui pour la plupart sont des mesures de mortalité sur des espèces d'invertébrés ou d'algues.

Cependant, la question de la signification écotoxicologique de la réponse biologique mesurée par un biomarqueur est débattue et reste un champ de recherche très ouvert (STEGEMAN et HAHN, 1994). En effet, s'il est vrai qu'un impact sur la population, hormis dans le cas d'une pollution accidentelle, est nécessairement précédé par une perturbation au niveau de quelques individus (MUNKITTRICK et MC CARTY, 1995), la réciproque est moins évidente : une perturbation de type biochimique n'entraîne pas nécessairement des effets irréversibles pour l'organisme ni, a fortiori, pour les populations. Cependant, tout stress entraîne une dépense énergétique pour l'organisme donc un coût, et, s'il se prolonge, il y a un risque non négligeable que d'autres effets apparaissent (sur la croissance, la reproduction...) (ADAMS et al., 1990 ; CALOW, 1991). 
Ainsi, dans l'état actuel des connaissances, les biomarqueurs renseignent davantage sur l'exposition des organismes à des contaminants (approche diagnostique) que sur l'évaluation d'un risque réel pour les populations (approche prédictive) (LAGADIC et al., 1998). Au fur et à mesure que la réponse mesurée gagne en pertinence écologique, elle perd en sensibilité, spécificité et précision pour la détection d'une exposition à un contaminant (RAPPORT et al., 1985 ; ADAMS et al., 1990). A contrario, plus les marqueurs sont sensibles et plus les risques de « faux positifs " sont élevés (CHAPMAN, 1991). Ainsi, il est par nature impossible d'obtenir simultanément une bonne sensibilité (en temps et en concentration) et une bonne représentativité écologique. Ces variables biologiques, au niveau de l'organisme ou de la population, sont donc complémentaires, chacune apportant sa part d'information sans remplacer l'autre (ATTRILL et DEPLEDGE, 1997).

Cette complémentarité est confirmée par des études récentes qui ont rassemblé sur le poisson des mesures de marqueurs biochimiques, de l'état de santé des individus (facteur de condition, état physiologique des organes, bilan parasitaire...) et de paramètres populationnels (KLOEPPER-SAMS et al, 1994 ; SCHLENK et al., 1996). II en ressort que si des corrélations existent entre les variables mesurées au niveau de l'individu, les liens entre des paramètres individuels et des paramètres populationnels sont quant à eux moins évidents. Ce type de constat n'est pas récent (PAYNE, 1984) et traduit la difficulté à lier des paramètres par nature éloignés. Plusieurs points permettent de l'expliquer.

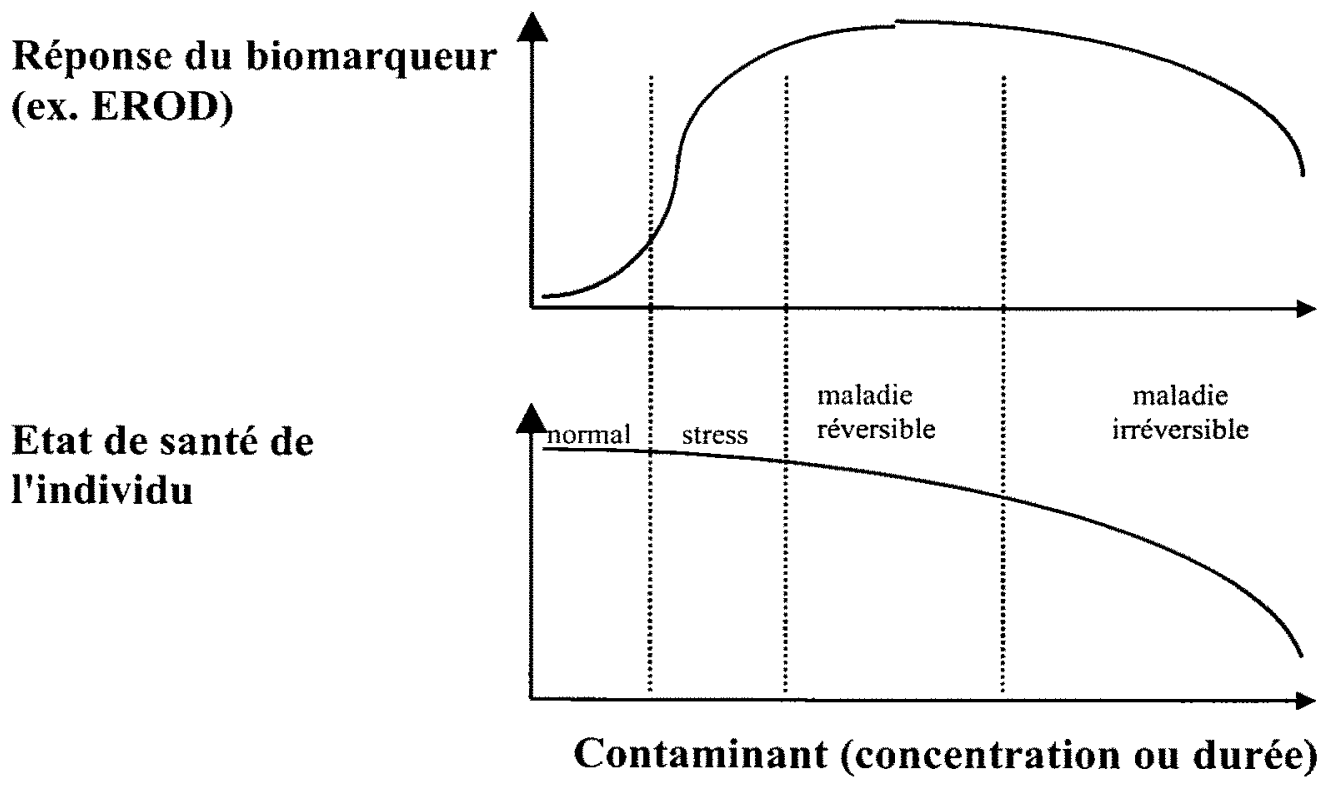

\section{Figure 1}

Schéma de réponse d'un biomarqueur (exemple de I'EROD) à la contamination (concentration ou durée) et comparaison avec l'état de santé (stress, maladie...) de l'individu (inspiré de MAYER et al., 1992 et de LAGADIC et al., 1997).

\section{Figure 1}

Biochemical response (e.g. EROD) and health (normal, stress, disease) of an organism exposed to a contaminant (concentration, time) (adapted from MAYER et al., 1992 and LAGADIC et al., 1997). 
D'une part, la moindre sensibilité des paramètres populationnels implique que le diagnostic qui en résulte témoigne généralement d'un impact important en terme d'intensité et/ou de durée, alors que parallèlement, pour de très fortes contaminations, les marqueurs biochimiques peuvent parfois présenter des réponses non spécifiques. Par exemple, lorsque l'organisme répond positivement et spécifiquement à la présence de certains contaminants par la synthèse accélérée d'une protéine (“induction "), il est possible que, pour de fortes concentrations, cette synthèse soit réduite (du fait de la toxicité qui s'exerce sur l'organisme) ce qui peut conduire à des faux négatifs (Figure 1). La Figure 1 illustre cette difficulté pour un marqueur biochimique à distinguer entre toxicité et réponse d'adaptation dans le cas précis du biomarqueur induction de l'activité enzymatique EROD que nous détaillerons plus loin. Certains auteurs ont tenu compte de cette toxicité potentielle pour interpréter des résultats dans des cas concrets d'études concernant le suivi des activités monooxygénases à cytochrome $P 450$ chez les poissons comme biomarqueur de pollution (LINDSTROM-SEPPA et OIKARI, 1990 ; VINDIMIAN et al., 1991).

D'autre part, les effectifs de populations de poissons, ou d'invertébrés, sont étroitement dépendants des conditions de débit, de substrat, etc... tandis que les biomarqueurs réagissent en premier lieu à la contamination tout en étant étroitement dépendants d'autres facteurs biologiques (âge, maturité sexuelle...). Cette non-linéarité de réponse entre effets précoces et effets intégrés ne peut que militer en faveur de la complémentarité entre biomarqueurs et bioindicateurs.

Dans une optique de détection de l'exposition des organismes à des contaminants et d'évaluation précoce de risques écotoxicologiques, le développement et la validation de biomarqueurs sont indispensables. Cependant, certains critères doivent être satisfaits et les étapes de validation doivent s'inscrire dans un ordre précis.

Ainsi, MAYER et al. (1992) ont rappelé les critères pour le choix d'un biomarqueur destiné à une utilisation sur le terrain :

1. mesure simple et économique ;

2. réponse dépendant simplement de la concentration et du temps et qui permette une quantification de l'exposition ou de l'effet à partir de la mesure du biomarqueur ;

3. bonne sensibilité ;

4. influence aux facteurs non toxiques (organisme, environnement, méthode) bien comprise et dans des limites acceptables ;

5. signification biologique (un impact sur « l'homéostasie " de l'individu ou de la population).

Ces conditions sont rarement satisfaites dans leur totalité pour les marqueurs biochimiques connus (DECAPRIO, 1997). En particulier, dans le cas de biomarqueurs dont la réponse est plus directement liée à la concentration des contaminants (biomarqueurs d'exposition), la condition 5 n'est pas remplie, même si on peut très bien imaginer que plus l'intensité du signal que représente le biomarqueur est élevée et plus le risque d'effets biologiques irréversibles est important. 
MC CARTHY (1990) définit cinq étapes de validation complète d'un biomarqueur :

1. collecte de données en sites de référence et en sites pollués (avec la prédominance d'un seul polluant) pour évaluer la capacité du biomarqueur à distinguer les situations polluées des situations non polluées;

2. expériences de laboratoire sur des espèces susceptibles d'être prélevées in situ pour quantifier les effets d'une exposition (mélange de polluants, effets long-terme...); liens avec d'autres biomarqueurs;

3. collecte de données sur des sites avec des situations complexes de pollution en utilisant plusieurs biomarqueurs ;

4. étude du pouvoir prédictif des biomarqueurs quant à des effets à des niveaux biologiques supérieurs (reproduction...) ;

5. prédiction des risques pour l'homme.

\section{EXEMPLES D'UTILISATION DE BIOMARQUEURS EN MILIEU AQUATIQUE}

Nous allons présenter successivement quelques exemples de biomarqueurs étudiés chez les poissons qui traduisent respectivement l'exposition des organismes à certaines familles de molécules : modulation d'activités enzymatiques, cassure de simple et de double brin d'ADN (effet génotoxique), perturbation de la synthèse de vitellogénine (altération de la reproduction).

\section{Mesure de l'induction de l'EROD}

Le biomarqueur qui a été le plus étudié jusqu'à présent chez le poisson est certainement l'induction du cytochrome P450 1A en particulier au niveau du tissu hépatique. Elle peut renseigner sur l'exposition des organismes à des polluants majeurs de l'environnement tels que les HAPs, PCBs, organochlorés.

Les premières études effectuées in situ datent des années 70 (PAYNE et PENROSE, 1975 ; PAYNE, 1976). L'induction peut être quantifiée en particulier par la mesure de l'activité monooxygénase EROD (EthoxyRésorufine-O-Dééthylase) catalysée spécifiquement par le cytochrome P450 1A. Un certain nombre d'études ont concerné les zones côtières américaines (STEGEMAN et al., 1987) ou françaises (LAFAURIE et al., 1989 ; GALGANI et al., 1991 ; NARBONNE et al., 1991). En milieu continental, les études ont été centrées sur la caractérisation de ces systèmes enzymatiques chez des poissons issus du milieu naturel et sur l'évaluation de l'impact de certains effluents d'usines (industrie chimique, papeteries, usines d'incinération de PCBs...) (MONOD et al., 1987 ; MONOD et al., 1988 ; LINDSTRÖM-SEPPÄ et OIKARI, 1988 ; MASFARAUD et al., 1990 ; VINDIMIAN et al., 1991 ; GARRIC et al., 1994 ; FLAMMARION, 1997). Après plusieurs années d'expérimentation, l'induction de l'activité EROD est actuellement envisagée comme outil de biosurveillance au sein du Réseau National d'Observation de la qualité du milieu marin, le RNO, par l'IFREMER. Au niveau européen, ce biomarqueur fait partie de la batterie de méthodes en cours de validation méthodologique dans le cadre du projet "BIOlogical MARkers of environmental contamination in marine ecosystem " (BIOMAR).

Des synthèses bibliographiques témoignent de l'étendue des études réalisées (PAYNE et al. 1987 ; GOKSØYR et FÖRLIN, 1992 ; STEGEMAN et al., 1992 ; BUCHELI et FENT, 1995 ; MONOD, 1997b ; FLAMMARION et al., 1998). Les connaissances accumulées sur ce biomarqueur montrent que les deux premières étapes définies cidessus par MC CARTHY (1990) ont été franchies. L'utilisation conjointe de plusieurs 
biomarqueurs, sensibles à différentes familles de contaminants, et de bioindicateurs sur des sites communs présentant des pollutions complexes permettrait d'atteindre le troisième objectif. Les objectifs 4 et 5 ne seront quant à eux probablement jamais atteints dans le cas du biomarqueur induction de l'EROD.

\section{Mesure de l'inhibition de l'activité acétylcholinestérasique}

La mesure de l'inhibition de l'activité AcétylCholinEstérasique (AChE) dans le muscle de poisson est un biomarqueur dont l'expression traduit spécifiquement l'exposition des poissons à des produits phytosanitaires de la famille des organophosphorés ou de celle des carbamates (BOCQUÉNÉ et al., 1990 ; BOCQUENE et al., 1993 ; PAYNE et al., 1996). L'inhibition est provoquée également, mais de manière non spécifique, par des contaminations métalliques. Cependant la connaissance exhaustive de tous les inhibiteurs potentiels de l'activité acétylcholinestérasique n'est pas encore acquise (PAYNE et al., 1996).

$\mathrm{Ce}$ biomarqueur a principalement été utilisé en milieu marin (GALGANI et BOCQUÉNÉ, 1998). Pour les poissons des milieux aquatiques continentaux, cette mesure s'est également révélée intéressante (RICHERT, 1994 ; PAYNE et al., 1996). L'inhibition observée chez les poissons d'une rivière du Beaujolais (RICHERT, 1994) a été vérifiée en laboratoire pour les concentrations mesurées in situ d'un organophosphoré (FLAMMARION et al., 1996).

On considère généralement que des inhibitions de l'ordre de $50 \%$ sont déjà susceptibles d'entraîner une mortalité à court terme (PAYNE et al., 1996) et on observe que les inhibitions sont significatives pour des expositions à des concentrations en contaminants proches de la toxicité létale (FLAMMARION et al., 1996). Ce biomarqueur n'est donc pas parmi les plus sensibles, mais en contrepartie l'inhibition est le signe objectif de désordres importants sur le comportement, la respiration (PAYNE et al., 1996). L'utilisation sur le terrain de ce biomarqueur a jusqu'à présent été peu répandue hormis en milieu marin et il manque certainement quelques efforts expérimentaux de terrain ou de laboratoire pour franchir les étapes de validation 1 et 2 définies par MC CARTHY (1990) (voir ci-dessus).

\section{Évaluation de l'impact génotoxique}

Si la mesure de marqueurs enzymatiques (induction de l'EROD, inhibition de l'AChE) peut apporter des informations sur le degré de pollution des milieux aquatiques et sur la biodisponibilité de certains polluants, elle n'est cependant pas suffisante pour évaluer des dommages significatifs au plan écotoxicologique au niveau des individus et des peuplements piscicoles exposés. Dans ce cadre, il est admis que le suivi de biomarqueurs d'effet des polluants, comme les marqueurs de génotoxicité, apportent des informations supplémentaires dans l'analyse des effets sur les organismes vivants (RETHER et al., 1997). En effet, la possibilité de mettre en évidence des altérations irréversibles du génôme animal représente un niveau pertinent de l'expression de la toxicité des polluants, eu égard aux désordres ultérieurs auxquels ils peuvent conduire lors d'étapes-clés comme la reproduction (ANDERSSON et WILD, 1994).

La métabolisation de xénobiotiques tels que les HAPs peut dans certaines situations donner naissance à des métabolites très réactifs susceptibles de se lier à l'ADN en formant des produits d'addition (adduits). La mesure de ces adduits à l'ADN, à la fois in vitro dans des cultures cellulaires ou ex vivo chez des poissons, a fait l'objet d'une littérature très abondante (DUNN et al., 1987 ; VARANASI et al., 1989a et b ; KURELEC et 
al., 1989 et 1991 ; MASFARAUD et al., 1992a et b ; VAN DER OOST et al., 1994). Parmi les techniques de mesure des adduits, la technique dite du post-marquage au ${ }^{32} \mathrm{P}$ est adaptée aux études de terrain. Elle est très sensible mais reste lourde de mise en œuvre (MASFARAUD et al., 1992a et b). L'essai comètes en conditions alcalines, plus accessible sur le plan expérimental, a été proposé récemment pour mettre en évidence l'effet génotoxique que représente l'apparition de cassures simple et double brin de l'ADN nucléaire de différents types de cellules (DEVAUX et al, 1997). Cette technique a franchi une première étape de validation sur le terrain en suivant limpact génotoxique des polluants chez les poissons de différentes stations de l'Ain et du Rhône présentant une contamination différenciée. Les résultats obtenus dans le cadre de l'étude de ce biomarqueur de génotoxicité ont permis de discriminer différentes stations sur le plan de la contamination par des molécules comme les PCBs et les métaux lourds, confortant ainsi les résultats de l'analyse chimique sur sédiments et muscles de poisson (DEVAUX et al, 1998). Des études de terrain actuellement en cours dans les bassins de la Moselle et du Rhône devraient permettre de progresser dans la validation de ce biomarqueur.

\section{Mesure de vitellogénine}

De nombreux composés, organochlorés (DDT, PCBs, etc...), ou des substances largement présentes dans les effluents de station d'épuration, comme par exemple les produits de dégradation de détergents alkylphénol polyéthoxylés ou les phtalates, ont été reconnus comme pouvant induire des perturbations du système endocrinien en induisant une activité oestrogène mimétique plus ou moins importante chez le poisson mâle (JOBLING et al., 1996; TYLER et al., 1996). Des études in situ, en aval de rejets de station d'épuration ont mis en évidence une telle activité oestrogénique chez des truites mâles exposées en aval des rejets, en relation avec une contamination du milieu par des alkylphénols (HARRIES et al., 1996 et 1997).

Pour détecter l'exposition des poissons à des composés de ce type, lors d'études in situ ou en laboratoire, il est possible de mesurer une lipoprotéine plasmatique, la ViTelloGénine (VTG), précurseur du vitellus de l'cuf du poisson et synthétisée par le fole au cours de la vitellogénèse. Cette augmentation de la concentration en vitellogénine, oestrogène-dépendante, n'a pas lieu chez les mâles ni chez les individus immatures, sauf dans les cas d'exposition à des substances oestrogéniques où les concentrations mesurées peuvent alors atteindre des niveaux équivalents à ceux mesurés chez des femelles matures. La mesure est réalisée par détection immunologique de la protéine sur du plasma sanguin prélevé sur les poissons exposés (TYLER et al., 1996 ; CHRISTIENSEN et al., 1997).

De nombreuses équipes se sont intéressées à l'utilisation de ce biomarqueur chez les poissons surtout depuis quelques années. Les études en cours visent essentiellement à préciser les substances susceptibles d'induire la synthèse de vitellogénine et à étudier les liens potentiels entre l'induction et des altérations au niveau des organes de reproduction, comme la présence d'ovocytes dans les testicules, ou des conséquences sur la différenciation sexuelle ou la fécondité des individus (JOBLING et al., 1998 ; FLAMMARION et al., 2000).

\section{Signification biologique des biomarqueurs présentés}

A l'occasion de l'interprétation des résultats obtenus avec les exemples de biomarqueurs présentés ci-dessus se pose systématiquement la question de la limite floue entre adaptation et toxicité. Autrement dit, est-ce que la réponse biochimique mesurée est le signe d'une réelle toxicité ou simplement une adaptation de l'organisme à un environnement pollué ? Cette discussion rejoint celle du compromis délicat entre 
sensibilité et signification écologique résumé dans un paragraphe précédent. Les exemples de l'EROD et de l'acétylcholinestérase illustrent respectivement un biomarqueur sensible signalant la réponse adaptative du poisson et un biomarqueur moins sensible mais dont la réponse significative est concomitante à l'apparition d'une toxicité avérée. Globalement les réponses des biomarqueurs présentés ici ne représentent pas per se un événement toxique pour l'organisme si les conséquences délétères de ces modifications biochimiques ne sont pas analysées au niveau de l'individu ou de la population. Les biomarqueurs représentant avant tout des systèmes d'alarme précoces de l'exposition des organismes à des polluants, II est alors nécessaire de rechercher les corrélations pouvant exister entre l'évolution de ces biomarqueurs et des désordres ultérieurs tels que par exemple l'apparition de maladies, de modifications au niveau populationnel («bottleneck", augmentation du taux moyen d'homozygotie), événements s'inscrivant dans une échelle de temps beaucoup plus large (MC CARTHY et SHUGART, 1990; SHUGART et THEODORAKIS, 1996). Cependant, nous rappelons que la nature multifactorielle de l'origine de ces événements rend généralement difficile la mise en évidence d'une relation de type causal. Selon BAYNE (1980) un effet biochimique d'un polluant est considéré comme significativement toxique s'il entraîne une réduction de valeur sélective. Mais cette définition suppose de pouvoir mesurer effectivement la valeur sélective et de faire la relation entre la réduction de valeur sélective et les modifications biochimiques enregistrées.

\section{Quelques informations pratiques}

Le tableau ci-dessous (Tableau I) fournit quelques éléments relatifs aux mesures de ces quatre biomarqueurs. Le matériel nécessaire est principalement un équipement classique de laboratoire de biochimie. Sur le terrain toutes ces techniques supposent cependant un échantillonnage des poissons par pêche électrique, ce qui peut nécessiter une sous-traitance ou un partenariat.

\section{Tableau I}

Quelques caractéristiques et modalités pratiques de quatre marqueurs biochimiques mesurés chez le poisson.

\section{Table 1}

General characteristics and protocols of four fish biochemical markers.

\begin{tabular}{lllll}
\hline & \multicolumn{1}{c}{ EROD } & \multicolumn{1}{c}{ AChE } & Essai des Comètes & \multicolumn{1}{c}{ VTG } \\
\hline Type de méthode & $\begin{array}{l}\text { activité } \\
\text { enzymatique }\end{array}$ & $\begin{array}{l}\text { activité } \\
\text { enzymatique }\end{array}$ & électrophorèse ADN & immunochimie \\
\hline Tissus & foie & muscle & cellules sanguines & sang (plasma) \\
\hline Conservation & azote liquide & azote liquide & $4^{\circ} \mathrm{C}$ en tube hépariné & $\begin{array}{l}\text { plasma conservé à } \\
-80^{\circ} \mathrm{C}\end{array}$ \\
\hline Equipement & $\begin{array}{l}\text { équipement de } \\
\text { biochimie, } \\
\text { fluorimètre } \\
\text { (au total 150kF) }\end{array}$ & $\begin{array}{l}\text { équipement de } \\
\text { biochimie, } \\
\text { colorimètre } \\
\text { (au total 100kF) }\end{array}$ & $\begin{array}{l}\text { électrophorèse, étuve, } \\
\text { microscope optique à } \\
\text { épifluorescence, } \\
\text { analyse d'images } \\
\text { (au total 200kF) }\end{array}$ & $\begin{array}{l}\text { équipement de } \\
\text { biochimie, } \\
\text { colorimètre } \\
\text { (au total 150kF) }\end{array}$ \\
\hline $\begin{array}{l}\text { Coút } \\
\text { approximatif* }\end{array}$ & 5F/poisson & 5F/poisson & 10F/poisson & 10F/poisson \\
\hline
\end{tabular}




\section{PROPOSITIONS D'OPTIMISATION DU SUIVI DE CES BIOMARQUEURS}

A court terme, quelques propositions concrètes peuvent être avancées pour améliorer et valider l'utilisation de ces biomarqueurs dans une perspective d'études en routine.

\section{Intégration dans un réseau de mesures}

II est délicat d'avancer un diagnostic fondé sur les résultats d'un seul site. II convient en effet de comparer ces résultats à ceux obtenus plus à l'amont ou plus à l'aval (références géographiques), ou encore par rapport à des mesures réalisées auparavant sur le même site (références temporelles). Le maximum d'information est donc obtenu dans le cadre d'un réseau dont les sites sont échantillonnés avec une fréquence compatible avec la variable biologique utilisée. Un réseau doit donc avoir une bonne couverture spatiale ainsi qu'une pérennité suffisante; et cela tant pour donner au gestionnaire une information sur la tendance évolutive (signe de bonne gestion ou d'une dégradation) que pour permettre aux scientifiques de mieux comprendre le fonctionnement du système étudié. Par exemple, l'échelle de temps compatible avec un suivi de l'EROD est de l'ordre du mois et l'échelle espace es: de l'ordre de la dizaine de kilomètres pour être cohérente avec la mobilité de certaines espèces de poissons. On peut en déduire le réseau et la fréquence correspondants. Mais compte tenu du coût d'un tel échantillonnage, il est possible de différencier les points à risque (effluents à étudier spécifiquement), des points de suivi qualité (type Réseau National de Bassins). Les seconds ne nécessitent qu'un suivi annuel pour évaluer les grandes tendances évolutives (dégradation ou restauration). A condition que les facteurs naturels de variabilité des biomarqueurs (par exemple espèce et maturité sexuelle des poissons pour l'EROD) soient bien pris en compte, cette accumulation de données routinières permettrait de dégager des tendances d'évolution spatiale et temporelle de la contamination tout en validant la qualité des biomarqueurs quant à la prédiction d'effets irréversibles à long terme.

L'utilisation d'une nouvelle variable biologique en routine dans un programme de biosurveillance doit être pensée de façon rigoureuse. Toutefois, certains biomarqueurs en sont à un point suffisant de maturité pour être intégrés dans un programme de routine. A petite échelle, la mesure de l'activité EROD sur trois espèces de cyprinidés a permis le suivi de sites peu pollués ou pollués dans le bassin du Rhône (FLAMMARION, 1997), confirmant les résultats d'études antérieures sur ce bassin fluvial (MONOD et al., 1988). La prise en compte des activités EROD en sites peu pollués ainsi que la quantification de l'influence de la maturité sexuelle des femelles a abouti à l'élaboration de classes d'induction EROD (de 1 à 5 selon la contamination du site) (FLAMMARION, 1997 ; FLAMMARION et GARRIC, 1999). A plus vaste échelle, un exemple nous est fourni par le RNO en milieu marin : après les premières années de mise en place, une harmonisation des conditions d'échantillonnage et de mesure de l'EROD a permis de suivre dans le temps et l'espace les sites choisis (LAGADIC et al., 1998). Dans la même optique, et pour favoriser l'acquisition d'une méthode de mesure commune aux différents laboratoires, il paraît indispensable d'engager dès à présent, des études de standardisation et de normalisation des méthodes (GOKSØYR et FÖRLIN, 1992). En ce qui concerne le dosage de l'activité EROD, plusieurs exercices d'intercomparaison ont été conduits entre laboratoires européens de l'Atlantique Nord et de la Méditerranée (STAGG et ADDISON, 1995 ; VAN DEN HEUVEL et al., 1995, VIARENGO et al., 2000). D'autres programmes sont en cours en particulier dans le cadre de la biosurveillance en milieu marin.

\section{Évaluation de niveaux de référence}

Les biomarqueurs sont soumis à des facteurs biologiques de variabilité (âge, maturité sexuelle, espèce, etc...) dont il s'agit de connaître le plus précisément possible les influences afin de faire la part de ce qui est réellement dû à la pollution. Cette 
connaissance passe entre autres par la recherche de valeurs de référence (en situation non polluée) (MAYER et al., 1992). Le choix des conditions de référence détermine la détection et la quantification d'un impact (NIXON et al., 1996). En ce qui concerne les biomarqueurs, peu d'études ont porté leur effort principal sur l'évaluation de valeurs de référence. Si cela a déjà été abordé dans le cas de l'induction de l'EROD (BALK et al., 1996 ; FLAMMARION et GARRIC, 1997), rien de tel n'a encore été publié sur les trois autres biomarqueurs dont il est question ici. Une des principales difficultés réside dans la quasi-impossibilité de trouver des zones exemptes de pollution et de toute trace d'activité humaine (AMIARD et al., 1998). Seule reste faisable l'établissement de références relatives obtenues dans les sites les moins pollués possible dont il s'agirait de suivre scrupuleusement les niveaux en contaminants.

\section{Étude de biomarqueurs chez d'autres organismes}

L'intégrité biologique des poissons est généralement considérée comme représentative de celle de l'écosystème dans sa globalité (ADAMS et al., 1993). Cependant les poissons, contrairement aux invertébrés, peuvent présenter une certaine mobilité. Pour tenter de pallier cette difficulté, on peut utiliser des poissons d'élevage placés dans des cages (FENET et al., 1996) ou dans des bassins expérimentaux de dérivation de l'effluent in situ (KOSMALA et al., 1998). Ces techniques présentent cependant d'autres limites méthodologiques. De ce fait, l'utilisation d'invertébrés est une piste intéressante pour l'avenir ; d'autant plus que la possibilité de mesurer un certain nombre de marqueurs biochimiques chez les invertébrés a déjà été confirmée depuis une dizaine d'années (VIARENGO et al., 1987 ; RIBERA et al., 1989 ; GARRIGUES et al., 1990 ; MALLEY et al., 1993 ; LIVINGSTONE et al., 1995 ; COSSU et al., 1997 ; ESCARTIN et PORTE, 1997 ; AMIARD-TRIQUET et al., 1998 ; MORA, 1998 ; PELLERINMASSICOTTE et TREMBLAY, 1998).

\section{CONCLUSION}

Actuellement, les gestionnaires soucieux de l'environnement disposent d'un certain nombre de marqueurs biochimiques de pollution plus ou moins validés. Ces biomarqueurs traduisent une réponse précoce de l'organisme exposé à des contaminants. Ils sont de fait un complément évident des programmes de surveillance chimique en traduisant un risque pour les organismes des écosystèmes aquatiques.

De par leur spécificité et leur précocité, les biomarqueurs sont des outils de gestion à fort potentiel, en particulier dans le cadre d'un diagnostic environnemental préalable à des investigations plus coûteuses en temps et en énergie, sans préjuger de la nature, en termes de toxicité ou d'adaptation, des informations qu'ils fournissent à l'observateur, et même si un travail important reste à faire sur leur valeur prédictive quant à des impacts au niveau des populations (MONOD, 1997a ; LAGADIC et al., 1998). Un diagnostic de la qualité des écosystèmes aquatiques doit reposer sur plusieurs variables biologiques complémentaires, car l'effet biologique n'est pas en général spécifique d'un seul contaminant. II apparaît que, dans l'état actuel des connaissances, il faille privilégier une combinaison à la fois de biomarqueurs et de bioindicateurs dont la complémentarité de diagnostics permette de déterminer en temps réel l'état de santé de l'organisme et des populations, de guider les efforts de restauration et d'évaluer les résultats de la gestion des milieux (VINDIMIAN et GARRIC, 1992 ; CORMIER et DANIEL, 1994). Un suivi à long terme de biomarqueurs, couplés à des mesures de perturbations écologiques, permettrait de conclure sur l'évaluation du risque écologique qui est potentiellement apportée par les biomarqueurs (LAGADIC et al., 1998). 
Afin de poursuivre leur validation, il s'agit dès à présent d'intégrer les biomarqueurs dans les réseaux de mesure en fonction de leur état de maturité (connaissances, intercalibration). Le prélèvement de poisson étant réalisé, il est judicieux d'étudier en parallèle plusieurs biomarqueurs afin d'augmenter la fiabilité du diagnostic, et de diminuer les coûts marginaux des différentes mesures. Lintégration des biomarqueurs dans un réseau de type Réseau Hydrobiologique et Piscicole rendrait ainsi possible la mesure d'une batterie de biomarqueurs à moindre coût en profitant des prélèvements de poissons réalisés en routine chaque année par le Conseil Supérieur de la Pêche. La mesure d'une batterie de variables biologiques est nécessaire car il n'existe pas de biomarqueur idéal, ni informatif sur toutes les molécules chimiques, ni prédictif quant aux effets à tous les niveaux d'intégration biologique.

\section{REMERCIEMENTS}

Ce travail a bénéficié des discussions fructueuses qui ont eu lieu dans le cadre à la fois du pilotage d'un travail de doctorat en Toxicologie de l'Environnement (FLAMMARION, 1997) et du projet de recherche REFEROD financé par le GIP Hydrosystèmes (Programme Variables Biologiques).

\section{BIBLIOGRAPHIE}

ADAMS S.M., BROWN A.M., GOEDE R.W., 1993. A quantitative health assessment index for rapid evaluation of fish condition in the field. Trans. Am. Fish. Soc., 122, 63-73.

ADAMS S.M., SHUGART L.R., SOUTHWORTH G.R., HINTON D.E., 1990. Application of bioindicators in assessing the health of fish populations experiencing contaminant stress. In "Biomarkers of environmental contamination ", MC CARTHY J.F., SHUGART L.R. (ed.), Lewis Publishers, Boca Raton, Floride, USA, p. 333-353.

AMIARD J.C., CAQUET Th., LAGADIC L., 1998. Les biomarqueurs parmi les méthodes d'évaluation de la qualité de l'environnement. In "Utilisation de biomarqueurs pour la surveillance de la qualité de l'environnement ", LAGADIC L., CAQUET T., AMIARD J.C., RAMADE F. (ed.), Tec et Doc Lavoisier, Paris, $X X|-X X X|$ (introduction).

AMIARD-TRIQUET C., RAINGLET F., LARROUX C., REGOLI F., HUMMEL H., 1998. Metallothioneins in arctic bivalves. Ecotoxicol. Environ. Saf., 41, 96-102.

ANDERSSON S.L., WILD G.C., 1994. Linking genotoxic responses and reproductive success in ecotoxicology. Environ. Heaith Perspect., 102, 9-12.

ATTRILL M.J., DEPLEDGE M.H., 1997. Community and population indicators of ecosystem health : targeting links between levels of biological organisation. Aquat. Toxicol., 38, 183-197.

BALK L., LARSSON A., FORLIN L., 1996. Baseline studies of biomarkers in the feral female perch (Perca fluviatilis) as tools in biological monitoring of anthropogenic substances. Mar. Environ. Res., 42, 203-208.

BAYNE B.L., 1980. Physiological measurements of stress. Rapp. R.V. Réun. Const. Int. Explor. Mer, 179, 56-61.

BENSON W.H., Di GIULIO R.T., 1992. Biomarkers in hazard assessments of contaminated sediments. In « Sediment toxicity assessment » BURTON G.A. (ed.), Lewis Publishers, Boca Raton, p. 241-265.

BOCQUENE G., GALGANI F., BURGEOT T., LE DEAN L., TRUQUET P., 1993. Acetylcholinesterase levels in marine organisms along french coasts. Mar. Pollut. Bull., 26 (2), 101-106. 
BOCQUENE G., GALGANI F., TRUQUET P., 1990. Characterization and assay conditions for use of AChE activity from several marine species in pollution monitoring. Mar. Environ. Res., 30, 75-89.

BUCHELI T.D., FENT K., 1995. Induction of cytochrome P450 as a biomarker for environmental contamination in aquatic ecosystems. Crit. Rev. Environ. Sci. Technol., 25 (3), 201-268.

CALOW P., 1991. Physiological costs of combating chemical toxicants : ecological implications. Comp. Biochem. Physiol., 100C, 3-6.

CHAPMAN P.M., 1991. Environmental quality criteria : what type should we be developing? Environ. Sci. Technol, 25 (8), 1353-1359.

CHRISTIENSEN L.B., PEDERSEN K.L., KORSGAARD B., BJERREGAARD P., 1997. Establishement of an ELISA for rainbow trout vitellogenin and field studies testing the oestrogenic effect of effluent from sewage treatment works. Ninth International Symposium on pollutant responses on marine organisms. (PRIMO 9). Bergen, Norway 27-30 april 1997.

CORMIER S.M., DANIEL B.F., 1994. Biomarkers : taking the science forward (ed.). Environ. Toxicol. Chem., 13 (7), 1011-1012.

COSSU C., DOYOTTE A., JACQUIN M.C., BABUT M., EXINGER A., VASSEUR P., 1997. Glutathion reductase, selenium-dependent glutathion peroxidase, glutathion levels, and lipid peroxidation in freshwater bivalves, Unio tumidus, as biomarkers of aquatic contamination in field studies. Ecotoxicol. Environ. Saf, 38, 122-131.

DECAPRIO A.P., 1997. Biomarkers : coming of age for environmental health and risk assessment. Environ. Sci. Technol., 31, 1837-1848.

DESCY J.P., COSTE M., 1988. Utilisation des diatomées benthiques pour l'évaluation de la qualité des eaux courantes. Contrat CEE B7123. FNDUP Namur/CEMAGREF Bordeaux, $49 \mathrm{p}$.

DEVAUX A., FLAMMARION P., BERNARDON V., GARRIC J., MONOD G., 1998. Monitoring of the chemical pollution of the river Rhône through measurement of DNA damage and cytochrome P4501A induction in chub (Leuciscus cephalus). Mar. Environ. Res., 46, 257-262.

DEVAUX A., PESONEN M., MONOD G., 1997. Alkaline Comet assay in rainbow trout hepatocytes. Toxicol. in vitro, 11, 71-79.

DUNN B.P., BLACK J.J., MACCUBBIN A., 1987. ${ }^{32}$ P-postlabeling analysis of aromatic DNA adducts in fish from polluted areas. Cancer Res., 47, 6543-6548.

DUTKA B.J., 1998. Foreword. In « Microscale testing in aquatic toxicology », WELLS P.G., LEE K., BLAISE C. (ed.), CRC Press, Boca Raton.

ESCARTIN E., PORTE C., 1997. The use of cholinesterase and carboxylesterase activities from mytilus galloprovincialis in pollution monitoring. Environ. Toxicol. Chem., 16, 2090-2095.

FENET H., CASELLAS C., BONTOUX J., 1996. Hepatic enzymatic activities of the European eel (Anguilla anguilla) as a tool for biomonitoring freshwater streams : laboratory and field caging studies. Wat. Sci. Technol., 33 (6), 321-329.

FLAMMARION P., 1997. Mesure in situ de l'induction du cytochrome P450 1A chez des cyprinidés d'eau douce. Optimisation de l'interprétation. Thèse de l'Université de Metz. Décembre 1997. 107 p. + annexes.

FLAMMARION P., BRION F., PALAZZI X., BABUT M., GARRIC J., MIGEON B., NOURY P., THYBAUD E., TYLER C.R., 2000. Estrogenic effects on chub (Leuciscus cephalus) : induction of vitellogenin and effects on the testicular structure. Ecotoxicology, 9, 127-135. 
FLAMMARION P., GARRIC J., 1997. Cyprinids EROD activities in low contaminated rivers : a relevant statistical approach to estimate reference levels for EROD biomarker? Chemosphere, 35 (10), 2375-2388.

FLAMMARION P., GARRIC J., 1999. A statistical approach for classifying the extent of EROD induction of fish sampled in clean and contaminated waters. Wat. Res., 33, 2683-2689.

FLAMMARION P., GARRIC J., MONOD G., 1998. Utilisation de l'activité enzymatique EROD chez les poissons des hydrosystèmes continentaux. In « Utilisation de biomarqueurs pour la surveillance de la qualité de l'environnement ", LAGADIC L., CAQUET T., AMIARD J.C., RAMADE F. (ed.), Tec et Doc Lavoisier, Paris, p. 57-75.

FLAMMARION P., MIGEON B., GARRIC J., 1996. Joint effects of copper sulphate and methidathion on rainbow trout (Oncorhynchus mykiss) EROD and AChE activities. Bull. Environ. Contam. Toxicol., 56, 3, 440-445.

GALGANI F., BOCQUENE G., 1998. Biomarqueurs moléculaires d'exposition des organismes marins aux pesticides organophosphorés et carbamates. In « Utilisation de biomarqueurs pour la surveillance de la qualité de l'environnement ", LAGADIC L., CAQUET T., AMIARD J.C., RAMADE F. (ed.), Tec et Doc Lavoisier, Paris, p. 111-134.

GALGANI F., BOCQUENE G., LUCON M., GRZEBYK D., LETROUIT D.F., CLAISSE D., 1991. EROD measurements in fish from the northwest part of France. Mar. Pollut. Bull., 22 (10), 494-500.

GARRIC J., BRAY M., MIGEON B., VOLLAT B., RICHERT C., 1994. Evaluation de l'écotoxicité de rejets industriels et urbains et de la contamination du milieu récepteur. Rapport CEMAGREF. $33 \mathrm{p}$.

GARRIGUES P., RAOUX. C., LEMAIRE P., MATHIEU A., RIBERA D., NARBONNE J.F., 1990. In situ correlations between polycyclic aromatic hydrocarbons (PAH) and PAH metabolizing system activities in mussels and fish in the mediterranean sea: preliminary results. Int. J. Environ. Anal. Chem., 38, 379-387.

GOKSØYR A., FÖRLIN L., 1992. The cytochrome P-450 system in fish, aquatic toxicology and environmental monitoring. Aquat. Toxicol., 22, 287-312.

HARRIES J.E., SHEAHAN D., JOBLING S., MATHIESSEN P., NEALL P., ROUTLEDGE E., RYCROFT R., SUMPTER J.P., TYLOR T., 1996. A survey of oestrogenic activity in United Kingdom inland waters. Environ. Toxicol. Chem., 15, 1993-2002.

HARRIES J.E., SHEAHAN D., JOBLING S., MATHIESSEN P., NEALL P., SUMPTER J.P., TYLOR T., ZAMAN N., 1997. Oestrogenic activity in five United Kingdom rivers detected by measurement of vitellogenesis in caged male trout. Environ. Toxicol. Chem., 16, 534-542.

HUGGETT R.J., KIMERLE R.A., MEHRLE P.M., BERGMAN H.L., 1992. "Biomarkers : biochemical, physiological and histological markers of anthropogenic stress ", SETAC Special Publications Series, Lewis Publishers, Chelsea, US, $347 \mathrm{p}$.

JOBLING S., NOLAN M., TYLER C.R., BRIGHTY G., SUMPTER J.P., 1998. Widespread sexual disruption in wild fish. Environ. Sci. Technol., 32, 2498-2506.

JOBLING S., SHEAHAN D., OSBORNE J.A., MATHIESSEN P., SUMPTER J.P., 1996. Inhibition of testicular growth in rainbow trout exposed to oestrogenic alkylphenol chemicals. Environ. Toxicol. Chem., 15, 194-202.

KLOEPPER-SAMS P.J., SWANSON S.M., MARCHANT T., SCHRYER R., OWENS J.W., 1994. Exposure of fish to biologically treated bleached-kraft effluent. 1. Biochemical, physiological and pathological assessment of Rocky mountain whitefish (Proposium williamsoni) and Longnose sucker (Catostomus catostomus). Environ. Toxicol. Chem., 13 (9), 1469-1482. 
KOSMALA A., MIGEON B., FLAMMARION P., GARRIC J., 1998. Impact assessment of a wastewater treatment plant using fish biomarker EROD : field and on-site experiments. Ecotoxicol. Environ. Saf., 41, 19-28.

KRAMER K.J.M., BOTTERWEG J., 1991. Aquatic biological early warning systems : an overview. In " bioindicators and environmental management ", JEFFREY D.W., MADDEN B. (ed.), Academic Press, London, p. 95-126.

KURELEC B., GARG A., KRCA S., CHACKO M., GUPTA R.C., 1989. Natural environment surpasses polluted environment in inducing DNA damage in fish. Carcinogenesis, $10(7), 1337-1339$.

KURELEC B., KRCA S., GARG A., GUPTA R.C., 1991. The potential of carp to bioactivate benzo(a)pyrene to metabolites that bind to DNA. Cancer Lett., 57, 255-260.

LAFAURIE M., GIUDICELLI J., CARRIERE S., LEMAIRE P., MATHIEU A., NEGRE Y., 1989. Pollutant biotransformation in marine teleost fish : use in environmental health evaluation. Adv. Appl. Biotechnol. Ser., 5, 141-152.

LAFONT M., 1989. Contribution à la gestion des eaux continentales : utilisation des oligochètes comme descripteurs de l'état biologique et du degré de pollution des eaux et des sédiments. Thèse Doct. Etat, Université Lyon I, 311 p. + annexes.

LAGADIC L., AMIARD J.C., CAQUET Th., 1998. Biomarqueurs et évaluation de l'impact écologique des polluants. In "Utilisation de biomarqueurs pour la surveillance de la qualité de l'environnement ", LAGADIC L., CAQUET T., AMIARD J.C., RAMADE F. (ed.), Tec et Doc Lavoisier, Paris, p. 299-307.

LAGADIC L., CAQUET T., AMIARD J.C., 1997. Biomarqueurs en écotoxicologie : principes et définitions. In "Biomarqueurs en écotoxicologie, Aspects fondamentaux ", LAGADIC L., CAQUET T., AMIARD J.C., RAMADE F. (ed.), Masson, Paris, p. 1-9.

LASCOMBE C., 1997. Les variables biologiques au service de la gestion des écosystèmes aquatiques. In séminaire national "Les variables biologiques: des indicateurs de l'état de santé des écosystèmes aquatiques "Ministère de l'Environnement, 2-3 Novembre 1994, CEMAGREF (Eds.), p. 27-37.

LEVEQUE C., 1997. Etat de santé des écosystèmes aquatiques : l'intérêt des variables biologiques. In séminaire national « Les variables biologiques : des indicateurs de l'état de santé des écosystèmes aquatiques "Ministère de l'Environnement, 2-3 Novembre 1994, CEMAGREF (Eds.), p. 13-26.

LINDSTROM-SEPPA P., OIKARI A., 1988. Hepatic xenobiotic bio-transformation in fishes exposed to pulpmill effluents. Wat. Sci. Technol., 20 (2), 167-170.

LINDSTROM-SEPPA P., OIKARI A., 1990. Biotransformation activities of feral fish in waters receiving bleached pulp mill effluents. Environ. Toxicol. Chem., 9, 14151424.

LIVINGSTONE D.R., 1993. Biotechnology and pollution monitoring : use of molecular biomarkers in the aquatic environment. J. Chem. Technol. Biotechnol, 57, 195-211.

LIVINGSTONE D.R., LEMAIRE P., MATTHEWS A., PETERS L.D., PORTE C., FITZPARTRICK P.J., FORLIN L., NASCI C., FOSSATO V., WOOTTON N., GOLDFARB P., 1995. Assessment of the impact of organic pollutants on goby (Zosterisessor ophiocephalus) and mussel (Mytilus galloprovincialis) from the Venice lagoon, Italy : biochemical studies. Mar. Environ. Res., 39, 235-240.

MALLEY D.F., KLAVERKAMP J.F., BROWN S.B., CHANG P.S.S., 1993. Increase in metallothionein in freshwater mussels Anadonta grandis exposed to cadmium in the laboratory and in the field. Water Pollut. Res. J. Can., 28, 253-273. 
MASFARAUD J.F., MONOD G., DEVAUX A., 1990. Use of the fish cytochrome P-450dependent 7-ethylresorufin $O$-deethylase activity as a biochemical indicator of water pollution. Study of the liver and the kidney of male and female nase (Chondrostoma nasus) from the river Rhône. Sci. Total Environ., 97-98, 729-738.

MASFARAUD J.F., DEVAUX A., PFOHL-LESZKOWICZ A., MALAVEILLE C., MONOD G., 1992a. DNA adduct formation and 7-ethoxyresorufin O-deethylase induction in primary culture of rainbow trout hepatocytes exposed to benzo(a)pyrene. Toxicol. In Vitro, 6, 523-531.

MASFARAUD J.F., PFOHL-LESZKOWICZ A., MALAVEILLE C., KEITH G., MONOD G.,1992b. 7-ethylresorufin O-deethylase activity and level of DNA-adducts in trout treated with benzo(a)pyrene. Mar. Environ. Res., 34, 351-354.

MAYER F.L., VERSTEEG D.J., MAC KEE M.J., FOLMAR L.C., GRANEY R.L., MAC CUME D.C., RATTNER B.A., 1992. Physiological and nonspecific biomarkers. In "Biomarkers : biochemical, physiological, and histological markers of anthropogenic stress ", HUGGETT R.J., KIMERLE R.A., MEHRLE P.M., BERGMAN H.L. (ed.), Lewis Publishers, Chelsea, Etats-Unis, p. 5-86.

MC CARTHY J.F., 1990. Concluding remarks : implementation of a biomarker-based environmental monitoring program. In "Biomarkers of environmental contamination ", MC CARTHY J.F., SHUGART L.R. (ed.), Lewis Publishers, Boca Raton, Floride, USA, p. 429-439.

MC CARTHY J.F., SHUGART L.R., 1990. «Biomarkers of environmental contamination ». Lewis Publishers, Boca Raton, Floride, USA.

MONOD G., DEVAUX A., RIVIERE J.L., 1987. Characterization of some monooxygenase activities and solubilization of hepatic cytochrome P-450 in two species of freshwater fish, the nase (Chondrostoma nasus) and the roach (Rutilus rutilus). Comp. Biochem. Physiol., 88C, 83-89.

MONOD G., DEVAUX A., RIVIERE J.L., 1988. Effects of chemical pollution on the activities of hepatic xenobiotic metabolizing enzymes in fish from the river Rhône. Sci. Total Environ., 73, 189-201.

MONOD G., 1997a. Les biomarqueurs d'exposition. In séminaire national " Les variables biologiques : des indicateurs de l'état de santé des écosystèmes aquatiques" Ministère de l'Environnement, 2-3 Novembre 1994, CEMAGREF (Eds.), 145-148.

MONOD G., 1997b. L'induction du cytochrome P4501A1. In « Biomarqueurs en écotoxicologie, Aspects fondamentaux ", LAGADIC L., CAQUET T., AMIARD J.C., RAMADE F. (ed.), Masson, Paris, p. 33-51.

MORA P., 1998. Caractérisation des cholinestérases de trois mollusques bivalves : Mytilus edulis, Mytilus galloprovincialis et Corbicula fluminea. Contribution au développement d'un biomarqueur de contamination des milieux marins et dulçaquicoles. Thèse de l'Université de Bordeaux I, $260 \mathrm{p}$.

MOUTHON J., 1993. Un indice biologique lacustre basé sur l'examen des peuplements de mollusques. Bull. Fr. Peche Piscic., 331, 397-406.

MUNKITTRICK K.R., MC CARTY L.S., 1995. An integrated approach to aquatic ecosystem health : top-down, bottom-up or middle-out? J. Aquat. Ecosyst. Health, $4,77-90$.

NARBONNE J.F., 1998. Historique-fondements biologiques de l'utilisation de biomarqueurs en écotoxicologie. In «Utilisation de biomarqueurs pour la surveillance de la qualité de l'environnement ", LAGADIC L., CAQUET T., AMIARD J.C., RAMADE F. (ed.), Tec et Doc Lavoisier, Paris, p. 1-7. 
NARBONNE J.F., GARRIGUES P., RIBERA D., RAOUX C., MATHIEU A., LEMAIRE P., SALAUN J.P., LAFAURIE M., 1991. Mixed-function oxygenase enzymes as tools for pollution monitoring : field studies on the French coast of the Mediterranean sea. Comp. Biochem. Physiol, 100C, 37-42.

NIXON S.C., MAINSTONE C.P., IVERSEN T.M., KRISTENSEN P., JEPPESEN E., FRIBERG N., PAPATHANASSIOU E., JENSEN A., PEDERSEN F., 1996. The harmonised monitoring and classification of ecological quality of surface waters in the European union. Final report. WRc Ref CO 4150. 289 p.

PAYNE J.F., 1976. Field evaluation of benzopyrene hydroxylase induction as a monitor for marine petroleum pollution. Science, 191, 945-946.

PAYNE J.F., 1984. Mixed-function oxygenases in biological monitoring programs : review of potential usage in different phyla of aquatic animals. In : "Ecotoxicological testing for the marine environment ", PERSOONE G., JASPERS E., CLAUS C. (ed.), State University Ghent and Inst. Mar. Scient. Res., Bredene, Belgium, Volume 1, p. 625-655.

PAYNE J.F., FANCEY L.L., RAHIMTULA A.D., PORTER E.L., 1987. Review and perspective on the use of mixed-function oxygenase enzymes in biological monitoring. Comp. Biochem. Physiol., 86, 233-245.

PAYNE J.F., MATHIEU A., MELVIN W., FANCEY L.L., 1996. Acetylcholinesterase, an old biomarker with a new future ? Field trials in association with two urban rivers and a paper mill in Newfoundland. Mar. Pollut. Bull., 32 (2), 225-231.

PAYNE J.F., PENROSE W.R., 1975. Induction of aryl hydrocarbon (Benzo [a] pyrene) hydroxylase in fish by petroleum. Bull. Environ. Contam. Toxicol., 14 (1), 112-116.

PELLERIN-MASSICOTTE J., TREMBLAY R., 1998. La fragilité lysosomale en tant que biomarqueur cytologique. In "Utilisation de biomarqueurs pour la surveillance de la qualité de l'environnement », LAGADIC L., CAQUET T., AMIARD J.C., RAMADE F. (ed.), Tec et Doc Lavoisier, Paris, p. 225-242.

RAPPORT D.J., REGIER H.A., HUTCHINSON T.C., 1985. Ecosystem behaviour under stress. Am. Nat., 125 (5), 617-640.

RETHER B., MASFARAUD J.F., KEITH G., DEVAUX A., MONOD G., 1997. Biomarqueurs de génotoxicité chez les végétaux et les animaux. In "Biomarqueurs en Ecotoxicologie-Aspects Fondamentaux ", LAGADIC L., CAQUET T., AMIARD J.C., RAMADE F. (ed.), Masson, Paris, p. 287-313.

RIBERA D., NARBONNE J.F., SUTEAU P., RAOUX C., GARRIGUES P., LAFAURIE M., 1989. Activities of the PAH metabolizing system in the mussel as a biochemical indicator for pollution : French coasts of the Mediterranean sea. Océanis, 15, 443449.

RICHERT C., 1994. Comparaison de marqueurs biochimiques chez les poissons et d'indicateurs écologiques pour le diagnostic in situ de la pollution toxique dans les cours d'eau. Thèse de l'Université Claude Bernard-Lyon I, $190 \mathrm{p}$.

SCHLENK D., PERKINS E.J., HAMILTON G., ZHANG Y.S., LAYHER W., 1996. Correlation of hepatic biomarkers with whole animal and population-community metrics. Can. J. Fish. Aquat. Sci., 53, 2299-2309.

SHUGART L.R., THEODORAKIS C., 1996. Genetic ecotoxicology : The genotypic diversity approach. Comp. Biochem. Physiol. 113C, 273-276.

STAGG R.M., ADDISON R.F., 1995. An inter-laboratory comparison of measurements of ethoxyresorufin O-deethylase activity in dab (Limanda limanda) liver. Mar. Environ. Res., 40, 93-108. 
STEGEMAN J.J., HAHN M.E., 1994. Biochemistry and molecular biology of monooxygenases : current perspectives on forms, functions, and regulation of cytochrome $\mathrm{P} 450$ in aquatic species. In "Aquatic toxicology : molecular, biochemical and cellular perspectives », MALINS D.C., OSTRANDER G.K. (ed.), Lewis Publishers, Chelsea, Etats-Unis, p. 235-335.

STEGEMAN J.J., TENG F.Y., SNOWBERGER E.A., 1987. Induced cytochrome P450 in winter flounder (Pseudopleuronectes americanus) from coastal Massachusetts evaluated by catalytic assay and monoclonal antibody probes. Can. J. Fish. Aquat. Sci., 44, 1270-1277.

STEGEMAN J.J., BROUWER M., DI GIULIO T., FORLIN L., FOWLER B.A., SANDERS B.M., VAN VELD P.A., 1992. Molecular responses to environmental contamination: enzyme and protein systems as indicators of chemical exposure and effect. In «Biomarkers : biochemical, physiological, and histological markers of anthropogenic stress ", HUGGETT R.J., KIMERLE R.A., MEHRLE P.M., BERGMAN H.L. (ed.), Lewis Publishers, Chelsea, Etats-Unis, p. 235-335.

TYLER C.R. , VAN DER EERDEN B., JOBLING S., PANTER G., SUMPTER J.P., 1996. Measurement of vitellogenin, a biomarker for exposure to oestrogenic chemicals, in a wide variety of cyprinid fish. J. Comp. Physiol. B, 166, 418-426.

VAN DEN HEUVEL M.R., MUNKITTRICK K.R., STEGEMAN J.J., DIXON D.G., 1995. Second-round interlaboratory comparison of hepatic ethoxyresorufin-O-deethylase activity in white sucker (Catostomus commersoni) exposed to bleached-kraft pulp mill effluent. Environ. Toxicol. Chem., 14 (9), 1513-1520.

VAN DER OOST R., VAN SCHOOTEN F.J., ARIESE F., HEIDA H., SATUMALAY K., VERMEULEN N.P.E., 1994. Bioaccumulation, biotransformation and DNA binding of PAHs in feral eel (Anguilla anguilla) exposed to polluted sediments : a field survey. Environ. Toxicol. Chem., 13, 859-870.

VARANASI U., REICHERT W.L., STEIN J.E., 1989a. ${ }^{32}$ P-postlabelling analysis of DNA adducts in liver of wild english sole (Parophrys vetulus) and winter flounder (Pseudopleuronectes americanus). Cancer Res., 49, 1171-1177.

VARANASI U., REICHERT W.L., LE EBERHART B.T., STEIN J.E., 1989b. Formation and persistence of benzo(a)pyrene-diolepoxyde-DNA adducts in liver of English sole (Parophrys vetulus). Chem. Biol. Interact., 69, 203-216.

VIARENGO A., LAFAURIE M., GABRIELIDES G.P., FABBRI R., MARRO A., ROMEO M., 2000. Critical evaluation of an intercalibration exercise undertaken in the framework of the MED POL biomonitoring program. Mar. Environ. Res., 49, 1-18.

VIARENGO A., MOORE M.N., MANCINELLI G., MAZZUCOTELLI A., PIPE R.K., FARRAR S.V., 1987. Metallothioneins and lysosomes in metal toxicity and accumulation in marine mussels : the effect of cadmium in the presence and absence of phenanthrene. Marine Biol. 94, 251-257.

VINDIMIAN E., GARRIC J., 1992. Bioessais et bioindicateurs de toxicité dans le milieu naturel. Ministère de l'environnement. Etude inter-agences $N^{\circ} 17,61 \mathrm{p}$.

VINDIMIAN E., NAMOUR P., MIGEON B., GARRIC J., 1991. In situ pollution induced cytochrome $\mathrm{P} 450$ activity of freshwater fish : barbel (Barbus barbus), chub (Leuciscus cephalus) and nase (Chondrostoma nasus). Aquat. Toxicol., 21, 255266. 